Repeated checking really does cause memory distrust

Behaviour Research and Therapy.

Adam S. Radomsky*, Philippe T. Gilchrist \& Dominique Dussault Concordia University

Keywords: Obsessive-compulsive disorder, compulsive checking, memory, metamemory, confidence, salience

*Corresponding author: Department of Psychology, Concordia University, 7141 Sherbrooke West, Montreal, QC, H4B 1R6, CANADA. adam.radomsky@concordia.ca 


\begin{abstract}
Memory phenomena associated with obsessive-compulsive disorder (OCD) have received increased attention in the recent literature. Some debate remains about whether OCD is characterized by deficits in memory per se, or by poor memory confidence. Following from a recent study that demonstrated memory distrust results from repeated checking of a virtual computerized stove, we asked 50 undergraduate students to repeatedly turn on, turn off and check either a real kitchen stove (relevant checking) or a real kitchen faucet (irrelevant checking) in a standardized, ritualized manner. All participants completed a final check of the stove following these 19 checking trials. Results indicated that following repeated relevant checking, participants reported significantly reduced memory confidence, vividness and detail. Repeated irrelevant checking did not produce these decreases. Results are discussed in terms of cognitivebehavioural formulations of OCD and in terms of the effects of repetition on memory and metamemory.
\end{abstract}




\section{Repeated checking really does cause memory distrust}

Recent cognitive-behavioural models of compulsive checking (Rachman, 2002; Salkovskis, 1999) have emphasized the influence of certain beliefs (e.g., inflated responsibility) on compulsive symptomatology. While concepts such as inflated responsibility, overestimation of threat and intolerance of uncertainty certainly play key roles in the phenomenology of OCD (Obsessive Compulsive Cognitions Working Group, OCCWG, 1997, 2001, 2003), there is now a growing emphasis on other forms of cognition (i.e., attention, memory, metamemory) associated with OCD. Unfortunately, much of this work lacks ecological validity or the specificity required to elucidate the cognitive phenomena of interest (Radomsky \& Rachman, 2004).

Recent ecologically valid investigations of memory confidence in association with OCD have been both provocative and informative. Tolin et al. (2001) conducted a study in which participants were asked to repeatedly recall groups of threat relevant and irrelevant objects. They found that while memory accuracy was not affected by this repetition, memory confidence decreased significantly over time. That is, repeated recollection resulted in diminished metamemory but had no detectable effects on memory itself.

In a study that was primarily an investigation of memory bias in association with compulsive checking, Radomsky, Rachman \& Hammond (2001) asked participants to complete checks of potentially threatening items in their homes under conditions of high, low and no responsibility and found a significant relationship between increased levels of responsibility and decreased confidence in memory. That is, as responsibility increased, memory confidence declined. Interestingly, these reductions in memory confidence were associated with increases in memory accuracy for threat-relevant information, suggesting that while threatening information 
may be better remembered by compulsive checkers, this positive memory bias may occur at the cost of a vivid, detailed and trusted memory.

More recently, van den Hout \& Kindt $(2003,2004)$ conducted a series of novel and elegant investigations attempting to elucidate the mechanism(s) responsible for decreased memory confidence in association with compulsive checking. In their research, van den Hout \& Kindt used a paradigm in which participants were asked to operate the gas rings on a virtual stove and to operate dimmer switches for virtual light bulbs. Specifically, participants were trained to use a computer program to operate simulated gas rings and light bulbs. While their experiments consisted of a number of variations, generally, one group of participants was asked to repeatedly check one set of items (i.e., the knobs on the stove) in what the authors termed "relevant checking", while another group was asked to repeatedly check the other set (i.e., the dimmer switches) in what the authors termed "irrelevant checking". Following these repeated checking trials, all participants were asked to complete one final check of the same set of items (i.e., the knobs on the stove) and were then asked a number of questions about their memory accuracy and metamemory (memory confidence, vividness and detail) for the most recent checking trial.

Results from their investigations revealed that non-clinical individuals who engaged in repeated relevant checking showed marked decreases in memory confidence, vividness and detail (van den Hout \& Kindt, 2003, 2004). In addition, these participants tended to shift from 'remembering' their most recent check at pre-test to simply 'knowing' that they had checked the relevant item at post-test. This change, based on a distinction made by Tulving (1985), shows that the source or overall quality of the memory had changed from something more episodic (i.e., 'remembering') to something more semantic (i.e., 'knowing'). Finally, van den Hout \& Kindt 
$(2003,2004)$ found no evidence of decreases in memory accuracy following repeated relevant checking and no evidence of decreases in metamemory variables following repeated irrelevant checking. Van den Hout and Kindt $(2003,2004)$ argued that while responsibility and other factors may lead someone to begin checking, it was the repetition involved that produced a shift from primarily perceptual processing to processing of a conceptual nature. This in turn led to reduced confidence, vividness and detail of memory, leaving accuracy unimpaired. These changes would likely produce increased urges to check and it was therefore proposed that checking leads to memory distrust, which leads to increased checking.

The findings of these elegant experimental investigations are consistent with a recently proposed cognitive model of compulsive checking (Rachman, 2002) in which it was proposed that inflated responsibility, perceived severity of harm and perceived probability of harm interact to produce checking behaviour, which is then maintained by a "self-perpetuating mechanism". While the studies by van den Hout \& Kindt $(2003,2004)$ described above certainly indicate that checking may well contribute to its own self-perpetuation, their protocols utilized 'virtual' objects and therefore did not involve any perceived threat resulting from participants' poor performance. Furthermore, while participants were responsible for their own performance during their checking of virtual gas rings and virtual light bulbs, there was likely little sense of responsibility for the prevention of harm in their investigations. Responsibility and threat estimation are two of the primary belief domains established by the Obsessive Compulsive Cognitions Working Group (OCCWG, 1997, 2001, 2003), and collectively account for more of the variance in OCD related beliefs than any other identified belief domain (OCCWG, in press). The studies of van den Hout \& Kindt $(2003,2004)$ would therefore likely benefit from replication and extension to include these key constructs of interest. 
Due to some of the limitations in previous work investigating aspects of cognition in clinical populations, an experimental study using a highly ecologically valid protocol is desirable (Radomsky \& Rachman, 2004). The limitations of previous studies can generally be characterized by low ecological validity, either because words were used as stimuli, or because the experimental protocols did not produce conditions in which the participants perceived their performance as meaningful and/or important. One way to build upon the extant work on repetition and metamemory, adding aspects associated with responsibility and threat perception, while also improving upon the ecological validity of a virtual checking task would be to conduct an experimental investigation of task repetition, memory and memory confidence in an environment conducive to perceived threats, such as a functioning kitchen. The current study attempts to achieve these goals under conditions that should promote both increased responsibility and a real perceived threat. It is expected that, under these conditions, repeated relevant checking should lead to reductions in memory confidence, vividness and detail, while repeated irrelevant checking should not. Based on previous findings (van den Hout \& Kindt, 2003, 2004), it was not expected that task repetition would lead to decreases in memory accuracy.

\section{$\underline{\text { Methods }}$}

\section{Measures}

Participants completed the Vancouver Obsessional Compulsive Inventory (VOCI: Thordarson, Radomsky, Rachman, Shafran, Sawchuk, \& Hakstian, 2004), the Beck Anxiety Inventory (BAI: Beck, \& Steer, 1990), and the Beck Depression Inventory II (BDI: Beck, Steer, 
\& Garbin, 1996). All of these measures have been shown to have acceptable psychometric properties.

Memory Accuracy. At both pre- and post- tests (see below) participants were given a diagram showing the six knobs on the stove. They were asked to mark three X's to indicate which three knobs they checked during the most recent trial.

Memory Confidence, Vividness and Detail. At both pre- and post- tests (see below) participants were asked to rate their confidence, vividness and detail of their memory for the most recent check on 100 point scales. Participants were told that a score of 0 represents "not at all" and that a score of 100 represents "extremely."

Confidence in outcome. At both pre- and post- tests (see below) participants were asked to indicate how confident they were (on a 0 to 100 scale) that all of the knobs were indeed off.

'Remember' vs. 'know'. At both pre- and post-tests, participants were asked to indicate whether their memory for the most recent trial was consistent with 'remembering' the event, or just 'knowing' that it happened. A description of the distinction was provided to them consistent with that proposed by Tulving (1985). Participants were told that:

"Knowing" that the knobs are all off means that you have a general sense that they are off. Even if you do not have a concrete memory, you just know they are turned off. For example, your memory of tying your shoes this morning is probably 'known' as opposed to 'remembered'. 
"Remembering" that the knobs are turned off means you can go through your memory and bring up a memory of the act (with specific features) of turning them off. For example, your memory of finding the lab in this building is probably 'remembered' as opposed to just 'known'.

\section{Participants}

Fifty-five undergraduate students from Concordia University participated in individual experimental sessions, although only fifty ( 9 males and 41 females) were included in the analyses (see below). Participation for this study was on a voluntary basis and participants were offered a non-alcoholic beverage as compensation for their time. On average, participants were $25.32(S D=7.47)$ years old and ranged in age from $19-49$ years. Scores on self-report measures were all within the normal range and are displayed in Table 1.

\section{Procedure}

Participants were tested individually in the laboratory's fully equipped kitchenette. Participants were randomly assigned to be trained on either the stove followed by the sink or the sink followed by the stove. All participants were trained to "open/turn-on", "close/turn-off" and "check" both the complete set of knobs on the stove and the knobs and faucet on the sink in a standardized, ritualized fashion. The stove used in this study was an electric stove. Throughout the training and testing on the stove, all of the plastic stove knobs were removed from the stove and participants were given one plastic knob which they were asked to use to operate all stove burners in the study. Furthermore, the burner lights on the stove were covered up so that there were no visual cues that could help participants to determine whether or not the stove was completely off following each check. Knobs on the stove were numbered one through six and 
the sink components and knobs were referred to as "hot" (to be set either to half or full), "cold" (to be set either to half or full) or the "tap" (to be set either to the left or to the right). Posted on the wall next to the stove was a diagram clearly indicating each stove knob by number to ensure that participants understood which number corresponded to which knob. During pilot testing, participants reported that the two sets of tasks (involving turning and wiggling the stove knobs or faucet in a standardized way) were equally difficult. The kitchen was dimly lit with the lighting fixed only on the stove and the sink. One participant was excluded from the study after stating that they did not understand the training provided and another was excluded because of difficulties with the English language.

Following the training phase and in order to increase perceived responsibility, participants were told that they would be left in the kitchenette by themselves for the next part of the study, and that communication with the experimenter would take place over an intercom affixed to a side wall in the kitchenette. After each instruction provided (“open/turn-on”, “close/turn-off" or "check"), participants were asked to walk back to the intercom to let the experimenter know that they had completed the task.

Once the participants were alone in the kitchenette, they were asked first to "turn-on", then "turn-off" and finally to "check" a set of three knobs on the stove according to the procedures that they had just learned. After these three tasks were completed, participants were guided out of the kitchen into a testing room where they provided pre-test ratings assessing memory confidence, vividness, and detail as well as a question to assess their memory accuracy (specifically, “which three knobs did you check?”), and a question about memory source ('remember' vs. 'know'). Participants scoring lower than 25 on any metamemory rating were 
excluded to ensure that following one trial, all participants had sufficient metamemory to recall their experiences clearly. (One participant was excluded for this reason.)

Next, participants were randomly assigned to either complete 19 sets of additional trials on the stove (relevant checking condition) or 19 sets of trials on the sink (irrelevant checking condition). Each trial was defined as including "turn-on", "turn-off" and "check" instructions given for a randomized set of three knobs or sink elements (e.g., "Please turn on knobs 2, 3 and 5."; "Please turn them off."; "Please check them."; "Please turn on the cold water to half way, the hot water to full and turn the faucet to the left."; "Please close them."; etc.). Following each instruction, the experimenter waited for the participant to complete each task before moving on to the next one. There were two randomized orderings of 19 sets of three knobs and two randomized orderings of 19 sets of three kitchen sink operations that were used to provide instructions to participants about which knobs or which aspects of the kitchen sink should be used. This was followed by a post-trial on the stove for all participants. All participants were then again guided to a testing room where they were asked to complete a post-test assessing memory and metamemory for this last trial on the stove, identical to the pre-test, but this time, specifically asking about their most recent (final) check of the stove. One participant was excluded from the relevant checking condition because the experimenters did not hear the participant checking the stove. (Stove checking produced audible 'clicks' which were easy to hear through a covered observation window.)

Lastly, all participants were asked to complete a questionnaire package including selfreport measures of symptoms of OCD (VOCI, Thordarson, et al., 2004), depression (BDI, Beck, Steer \& Garbin, 1996) and anxiety (BAI, Beck \& Steer, 1990). One participant was excluded because of missing data. Participants were then debriefed from the study. 


\section{$\underline{\text { Results }}$}

Mean comparisons of the scores on the questionnaires revealed no significant differences between conditions (all $p$ 's $>.05$ ), indicating acceptable random assignment. The questionnaires were therefore not included in the analyses as covariates. Means for the questionnaires, including the VOCI checking subscale, are included in Table 1. Finally, there were no significant differences in age or gender across groups.

\section{Memory \& Metamemory}

Memory accuracy and metamemory results are presented in Table 2 and Figure 1.

\section{Memory Accuracy}

Memory accuracy was assessed by scoring the number of correct responses to the question, "On the last checking trial you completed, which three knobs did you check?". Accuracy was extremely high for both conditions at pre- and post-tests. At pre-test, three relevant and two irrelevant checking participants remembered all but one knob correctly whereas all other participants had perfect accuracy scores. At post-test, there were five relevant checking participants who remembered all but one knob correctly, whereas all participants in the irrelevant condition obtained a perfect score. A $2 \times 2$ repeated measures ANOVA revealed that there were no significant changes in memory accuracy from pre- to post-test, $\mathrm{F}_{(1,48)}<1$, n.s., no significant differences in memory between the two conditions, although a trend towards reduced memory accuracy in the relevant checking condition was found, $\mathrm{F}_{(1,48)}=3.72, p<0.10$, and no significant interaction between time and condition $\mathrm{F}_{(1,48)}=2.00$, n.s. Because of the memory accuracy trend, and because all participants in the irrelevant checking condition obtained a perfect memory score 
at post-test, a follow-up Z-test was conducted and revealed that the relevant checking condition indeed showed a memory score that was significantly less than perfect (i.e., less than 3.0) at posttest, $Z=2.44, p<0.05$, although the size of the post-test difference between conditions (a mean difference of 0.2 out of 3.0) was small.

Memory confidence, vividness and detail

A 2x2 repeated measures MANOVA with confidence, vividness and detail as dependant variables, revealed that measures of metamemory significantly decreased from pre-test to posttest, $\mathrm{F}_{(1,48)}=12.87, p<0.001$, but that this change was moderated by a significant interaction between time and condition, $\mathrm{F}_{(1,48)}=8.55, p<0.005$. Planned comparisons between conditions at post-test, correcting for unequal variances where appropriate, revealed that the relevant checking condition reported significantly less confidence in memory $t_{(25.43)}=3.25, p<0.003$, significantly less memory vividness $t_{(29.07)}=3.47, p<0.002$, and significantly less detail in memory $t_{(39.57)}=3.14$, $p<0.003$, than the irrelevant checking condition. There were no significant differences between conditions on any metamemory variable at pre-test, all p's $>0.05$.

\section{Confidence in outcome}

A 2x2 repeated measures ANOVA revealed that there were no significant main effects for group, $\mathrm{F}_{(1,48)}<1$, for time $\mathrm{F}_{(1,48)}=1.58$, n.s. or for the interaction between group and time $\mathrm{F}_{(1,48)}=1.32$, n.s., indicating that participants were generally highly confident that all stove knobs had indeed been correctly turned off at pre- and post-test, regardless of condition.

'Remember' vs. 'know' 
A frequency chart of remember and know responses at post-test by condition is displayed in Figure 2. While there were no differences between groups in the frequency of remember vs. no responses at pre-test $\chi_{(1)}^{2}=1.22$, n.s., at post-test, significantly more participants in the relevant checking condition reported only 'knowing' which knobs they checked, compared with the irrelevant checking condition, in which participants primarily 'remembered' which knobs they checked $\chi_{(1)}^{2}=4.16, p<0.05$. That is, the source and quality of memory were generally reduced in the relevant checking condition but not in the irrelevant checking condition.

\section{$\underline{\text { Discussion }}$}

The results of the current investigation demonstrate that repeated relevant checking does indeed lead to memory distrust. That is, when participants were asked to repeatedly check a stove, their memory confidence, vividness and detail significantly declined both compared to pre-test scores following only one check of the stove, and compared to a group of participants who were asked to engage in repeated irrelevant checking of a kitchen faucet. These results are consistent with the findings of van den Hout \& Kindt $(2003,2004)$, and lead one to conclude that the incorporation of elevated threat (both in terms of probability and severity of harm) as well as conditions that promote increased responsibility do not inhibit the significant declines in metamemory (confidence, vividness and detail) following repeated relevant checking.

Interestingly, the results suggest that repeated relevant checking under ecologically valid conditions may produce very small but significant declines in memory accuracy. While this contradicts the findings of van den Hout \& Kindt (2003, 2004), and may not be consistent with previous findings of memory bias in OCD (Radomsky \& Rachman, 1999; Radomsky, Rachman 
\& Hammond, 2001), it raises a set of interesting questions about the relationships between memory and metamemory in association with this particular task. It could be that the significance of the difference in memory accuracy scores is simply amplified by the complete absence of variance in the perfect scores obtained by all participants in the irrelevant checking group. However, it could be that repeated checking under conditions that were designed to promote high perceived responsibility and threat perceptions results in not only memory distrust, but also in slight decrements in memory accuracy. Furthermore, if repeated checking in this paradigm does indeed lead to small reductions in memory accuracy, it would be interesting to learn what specific psychological and/or environmental factors contribute to these reductions.

It should also be noted that the reductions in metamemory found in the current investigation appear to be less robust than those found by van den Hout and Kindt $(2003,2004)$. This could well be accounted for by the fact that completing checks of virtual objects is certainly less of a vivid and detailed experience than completing checks of real objects in an actual kitchen. Van den Hout and Kindt $(2003,2004)$ proposed that repeated checking promotes a shift from primarily perceptual processing that occurs during early checks to primarily conceptual processing that occurs after repeated checks. This shift is hypothesized to reduce memory vividness and detail, which in turn reduce memory confidence. Given that an in vivo checking task likely provides a richer encoding environment with much more information to perceive than in a virtual checking task, it would be expected that reductions in these aspects of metamemory would likely be much slower. It could also be argued however that increases in perceived threat and responsibility could contribute to greater reductions in metamemory, but it is proposed that rather, they would likely contribute to increased urges to check in a clinical sample. These are empirical questions and would benefit from replication and further investigation. 
Of course, there are some limitations to the current experimental investigation. In this study, all irrelevant checking was conducted on the sink and all relevant checking was carried out on the stove. Without continued checking, both objects would require a fairly long wait in the lab in order to determine that they were indeed properly shut off (for the stove, to wait until the burners cooled down and for the sink, to wait to ensure that there were no slow leaks), but there are fundamental differences about the nature of the threats presented by each object and this could be confounded with the relevant vs. irrelevant checking conditions in this investigation. While it might be interesting to counterbalance this in a subsequent study, we chose not to, partially due to considerations of power and also because van den Hout \& Kindt (2003) did counterbalance their relevant and irrelevant checking conditions in at least one study and found no differences between the metamemory of participants who engaged in relevant stove checking and those who engaged in relevant light bulb checking. As such, it is highly unlikely that this addition would have produced a different pattern of results. However, it would be quite interesting to investigate the differences between repeated checking of something that could indeed lead to some kind of disaster occurring (e.g., a functioning electric stove) and repeated checking of something that could not (e.g., an electric stove that was not plugged in to the electrical outlet).

In addition, no measures of perceived threat or perceived responsibility associated with the task were taken during the experiment. While it would certainly be interesting and useful to do this, we are confident that even though there were some factors that may have resulted in less than maximal perceived threat and responsibility (e.g., an experimenter in the next room), perceived threat and responsibility in this investigation were almost certainly higher than what would have been the case in a virtual checking task where there were obviously no real 
consequences of checking improperly. Furthermore, the heat generated by the stove burners and the fact that participants were completely alone and unobserved while checking in the testing room would almost certainly have elevated perceived threat and responsibility associated with the experimental task.

One particularly interesting observation made during the testing of participants was the amount of frustration they appeared to display during the protocol. Several participants asked the experimenter to tell them how many more checks there would be in both the relevant and irrelevant checking conditions. (We provided a standardized response that they were doing well, but that we couldn't tell them for how much longer they would be checking.) During debriefing, other participants described the experiment as both "frustrating" and "annoying". While patients who engage in compulsive checking often report frustration with their rituals, their primary emotion during checking is more likely to be anxiety. In future investigations of this type, it would be interesting and useful to assess frustration and anger in the context of repeated checking to ascertain the effects of checking on a variety of emotional experiences.

The results of this investigation help to shed light on the self-perpetuating mechanism proposed by Rachman (2002) in a recent cognitive model of compulsive checking. It appears as if mere repetition in the presence of perceived responsibility and perceived threat has dramatic effects on metamemory and possibly subtle effects on memory accuracy. While this finding does not speak to the possible influences of repetition on perceived responsibility, seriousness of harm and probability of harm, it does demonstrate the effects of these multipliers (Rachman, 2002) and repetition on confidence and other metamemory variables associated with the final state of the checked object. While clinicians have known for years that checking behaviour is counterproductive, there is now some empirical evidence for why it is counterproductive. It 
leads to memory distrust - the very thing it is hoped to diminish. This in turn promotes continued and/or renewed checking, paradoxically leading to even less memory confidence, vividness and detail.

These initial findings lead one to speculate about possible factors that might be involved in the mechanism(s) associated with these reductions in metamemory. Other experimental investigations examining attention focus during repeated checking, the amount of checking necessary to produce significant metamemory decreases and possible treatment components that could attenuate these decreases are currently underway and should lead to additional information about the processes involved, as well as possible practical applications toward the treatment of compulsive checking. There are of course, several clinical applications of the findings in the current investigation. Psychoeducation about the effects of repeated checking would likely be very helpful in motivating patients to reduce their checking behaviour. While it has been known for many years that exposure and response prevention can be an effective treatment for OCD (Meyer, 1966), the results of the current experiment show how response prevention might improve memory and memory confidence for checked objects. Finally, the possibilities associated with behavioural experiments used in treatment designed to assess the impact of repetition on metamemory are considerable, and results from this experimental investigation will hopefully lead to the increased incorporation of such exercises in treatment.

Finally, this study represents an effort to investigate cognitive phenomena associated with a specific manifestation of OCD, namely compulsive checking. While similar phenomena may operate in other forms of OCD, such as repeated washing rituals, compulsive list-making or counting, the checking-specific nature of the experiment is consistent with a recent call for "subtype-specific research" made by members of the Obsessive-Compulsive Cognitions 
Working Group (McKay, et al., 2004; Radomsky \& Taylor, in press). It is proposed that investigations exploring aspects of specific types of OCD, rather than OCD in general, will be most helpful in elucidating cognitive phenomena of particular importance to each symptom type. These investigations already have been particularly fruitful in the past and have led to the development of specific cognitive-behavioural models of obsessions (Rachman, 1997, 1998), compulsive hoarding (Frost \& Hartl, 1996), compulsive checking (Rachman, 2002), and most recently of compulsive cleaning and washing (Rachman, 2004). It is proposed that experimental investigations of specific manifestations of OCD will be most fruitful in elucidating important cognitive phenomena with direct applications to both assessment procedures and treatment interventions. 
$\underline{\text { Author Notes }}$

We are grateful to Sarah Brown Tesolin for her help with participant scheduling and laboratory coordination and to the anonymous reviewers for their helpful comments. Portions of this data were presented at the 2003 Association for the Advancement of Behavior Therapy conference in Boston, MA and at the 2004 European Association of Behavioural and Cognitive Therapies conference in Manchester, UK. This study was supported by a research operating grant from the Natural Sciences and Engineering Research Council of Canada (NSERC, \#RGPIN-2498332002) awarded to the first author. 


\section{$\underline{\text { References }}$}

Beck, A.T., \& Steer, R.A. (1990). Beck Anxiety Inventory Manual. Toronto: Psychological Corporation.

Frost, R. O., \& Hartl, T. L. (1996). A cognitive-behavioral model of compulsive checking. Behaviour Research and Therapy, 34, 341-350.

Beck, A.T., Steer, R.A., \& Garbin, M.G. (1996). Beck Depression Inventory Manual (2 $2^{\text {nd }}$ ed.). San Antonio: Psychological Corporation.

McKay, D., Abramowitz, J.S., Calamari, J.E., Kyrios, M., Radomsky, A.S., Sookman, D., Taylor, S., \& Wilhelm, S. (2004). A Critical Evaluation of Obsessive-Compulsive Disorder Subtypes: Symptoms versus Mechanisms. Clinical Psychology Review, 24(3), 283-313.

Obsessive Compulsive Cognitions Working Group. (in press). Psychometric validation of the Obsessive Beliefs Questionnaire and the Interpretation of Intrusions Inventory: Part 2, factor analyses and testing of a brief version. Behaviour Research and Therapy.

Obsessive Compulsive Cognitions Working Group. (1997). Cognitive assessment of obsessivecompulsive disorder. Behaviour Research and Therapy, 35(7), 667-681.

Obsessive Compulsive Cognitions Working Group. (2001). Development and initial validation of the Obsessive Beliefs Questionnaire and the Interpretation of Intrusions Inventory. Behaviour Research and Therapy, 39, 987-1006.

Obsessive Compulsive Cognitions Working Group. (2003). Psychometric validation of the Obsessive Beliefs Questionnaire and the Interpretation of Intrusions Inventory: Part I. Behaviour Research and Therapy, 41(8), 863-878. 
Rachman, S. (2004). Fear of contamination. Behaviour Research and Therapy, 42(11), 1227 1255.

Rachman, S. (1997). A cognitive theory of obsessions. Behaviour Research and Therapy, 35, 793-802.

Rachman, S. (1998). A cognitive theory of obsessions: Elaborations. Behaviour Research and Therapy, 36, 385-401.

Rachman, S. (2002). A cognitive theory of compulsive checking. Behaviour Research and Therapy, 40(6), 624-639.

Radomsky, A.S., \& Rachman, S. (2004). The importance of importance in OCD memory research. Journal of Behavior Therapy and Experimental Psychiatry, 35(2), 137-151.

Radomsky, A. S., \& Rachman, S. (1999). Memory bias in obsessive-compulsive disorder (OCD). Behaviour Research and Therapy, 37, 605-618.

Radomsky, A.S., Rachman, S., \& Hammond, D. (2001). Memory bias, confidence and responsibility in compulsive checking. Behaviour Research \& Therapy, 39(7), 813-822.

Radomsky, A.S., \& Taylor, S. (in press). Subtyping OCD: Prospects and problems. Behavior Therapy.

Salkovskis, P.M. (1999). Understanding and treating obsessive-compulsive disorder. Behaviour Research and Therapy, 37(suppl. 1), S29-S52.

Salkovskis, P.M., Shafran, R., Rachman, S., \& Freeston, M.H. (1999). Multiple pathways to inflated responsibility beliefs in obsessional problems: Possible origins and implications for therapy and research. Behaviour Research and Therapy, 37(11), 1055-1072. 
Thordarson, D.S., Radomsky, A.S., Rachman, S., Shafran, R., Sawchuk, C.N., \& Hakstian, A.R. (2004). The Vancouver Obsessional Compulsive Inventory (VOCI). Behaviour Research \& Therapy, 42(11), 1289-1314.

Tolin, D.F., Abramowitz, J.S., Brigidi, B.D., Amir, N., Street, G.P., \& Foa, E.B. (2001). Memory and memory confidence in obsessive-compulsive disorder. Behaviour Research and Therapy,39(8), 913-927.

Tulving, E. (1985). Memory and consciousness. Canadian Psychology, 26(1), 1-12.

Van den Hout, M. \& Kindt, M. (2003). Repeated checking causes memory distrust. Behaviour Research and Therapy, 41(3), 301-316.

Van den Hout, M. \& Kindt, M. (2004). Obsessive-compulsive disorder and the paradoxical effects of perseverative behaviour on experienced uncertainty. Journal of Behavior Therapy and Experimental Psychiatry, 35(2), 165-181. 
Table 1 - Participant Characteristics

\begin{tabular}{lllll}
\hline Condition & VOCItotal & VOCIcheck & BAI & BDI \\
\hline $\begin{array}{llll}\text { Irrelevant Checking } \\
(\mathrm{n}=25)\end{array}$ & $\begin{array}{l}15.38 \\
(17.90)\end{array}$ & $\begin{array}{l}0.76 \\
(2.09)\end{array}$ & $\begin{array}{l}7.88 \\
(7.67)\end{array}$ & $\begin{array}{l}6.32 \\
(5.82)\end{array}$ \\
$\begin{array}{llll}\text { Relevant Checking } \\
(\mathrm{n}=25)\end{array}$ & 20.13 & 1.58 & 8.29 & 6.35 \\
& $(19.77)$ & $(2.64)$ & $(5.47)$ & $(6.13)$ \\
\hline $\begin{array}{l}\text { Total } \\
(\mathrm{n}=50)\end{array}$ & 17.70 & 1.16 & 8.08 & 6.33 \\
\hline
\end{tabular}

Note: Groups did not differ on any measure (all p’s $>0.05$ ). 
Table 2 - Memory and metamemory pre- and post- repeated checking

\begin{tabular}{lcc|ccc}
\hline & \multicolumn{2}{c}{ Pre-test } & \multicolumn{2}{c}{ Post-test } \\
\cline { 2 - 6 } & $\begin{array}{l}\text { Irrelevant } \\
\text { checking }\end{array}$ & $\begin{array}{c}\text { Relevant } \\
\text { checking }\end{array}$ & $\begin{array}{l}\text { Irrelevant } \\
\text { checking }\end{array}$ & $\begin{array}{c}\text { Relevant } \\
\text { checking }\end{array}$ \\
\hline Memory Accuracy (\# of knobs correctly recalled) & 2.92 & 2.88 & 3.00 & 2.80 \\
& $(0.28)$ & $(0.33)$ & $(0.00)$ & $(0.41)$ \\
Confidence in memory of specific knobs checked & 97.16 & 95.28 & 98.16 & 80.00 \\
in most recent trial & $(6.29)$ & $(9.85)$ & $(4.75)$ & $(27.54)$ \\
Vividness of memory for most recent trial & 96.12 & 89.56 & 94.56 & 74.00 \\
& $(6.62)$ & $(16.30)$ & $(9.21)$ & $(28.17)$ \\
Detail in memory for most recent trial & 94.20 & 89.28 & 89.56 & 69.00 \\
& $(10.28)$ & $(18.54)$ & $(16.99)$ & $(27.99)$ \\
Confidence that all knobs are off & & & & \\
& 96.00 & 96.52 & 97.80 & 96.60 \\
& $(8.04)$ & $(8.23)$ & $(5.22)$ & $(10.48)$ \\
\hline
\end{tabular}


Figure 1 - Metamemory and memory scores pre- and post- repeated checking

\section{Confidence}

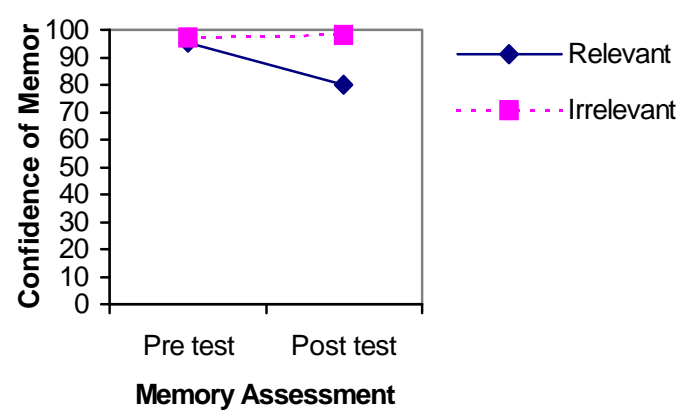

Detail

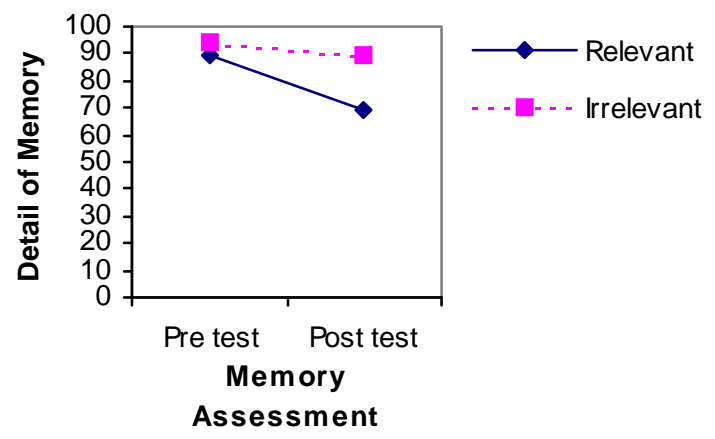

Vividness

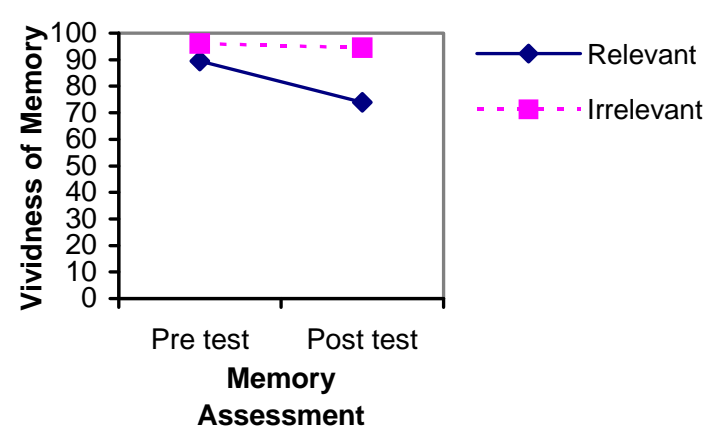

Accuracy

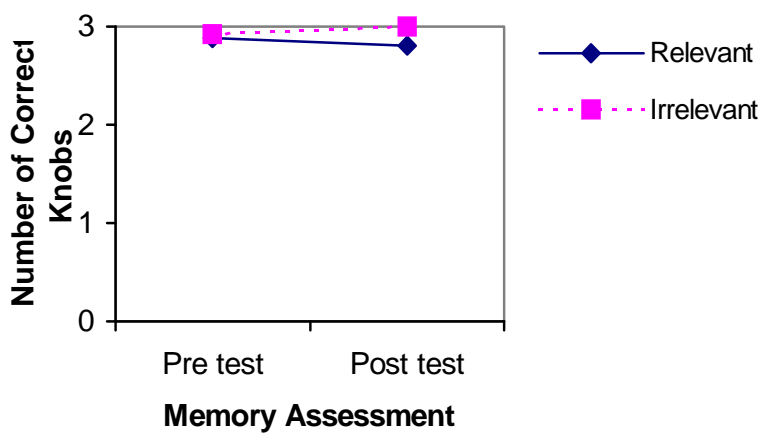


Figure 2 - Number of participants reporting 'Remember' vs. 'Know' at Post-Test

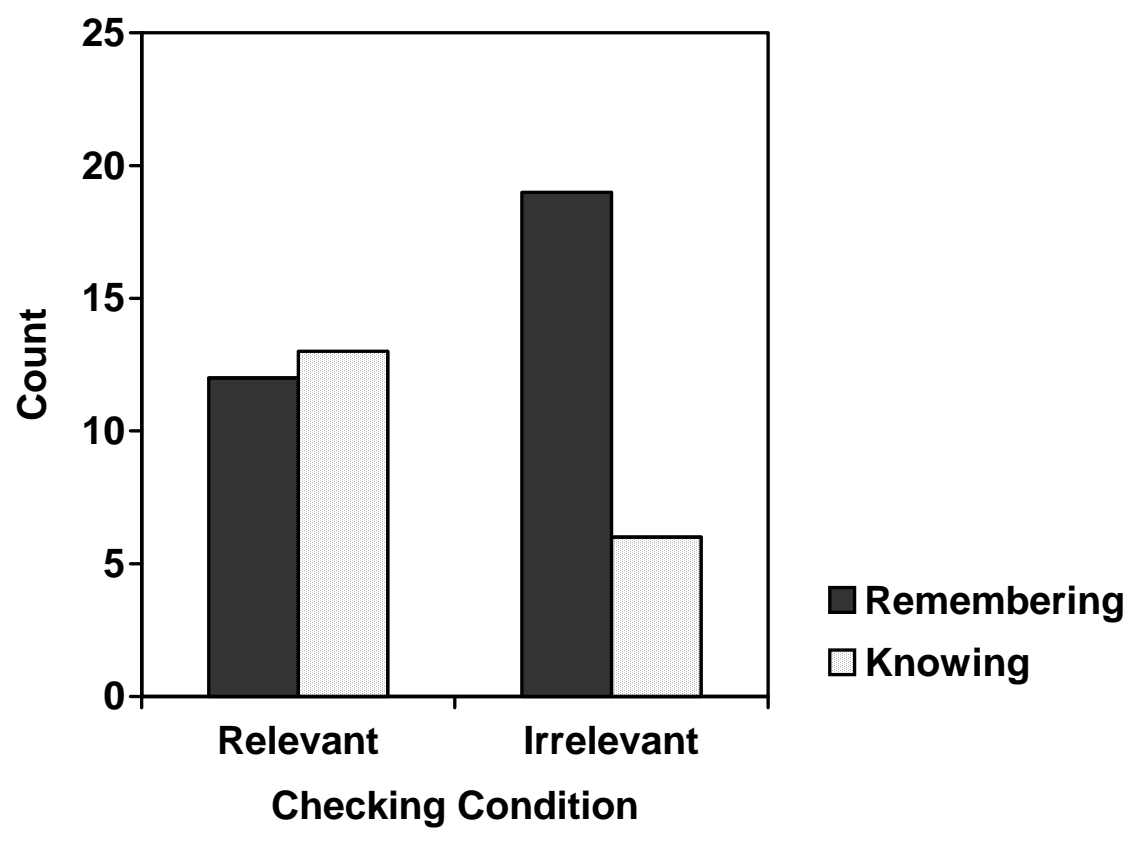

